The suggestion is also made that $\mathrm{CO}_{2}$ may be strongly dissociated by the metastable $\mathrm{Xe}$ atoms (and $\mathrm{H}_{2} \mathrm{O}$ somewhat less strongly), thus producing oxygen atoms which combine to form the $\mathrm{O}_{2}$ molecules; $\left(\mathrm{D}\left(\mathrm{CO}_{2}\right)=5.5 \mathrm{v}\right.$, $\left.\mathrm{D}\left(\mathrm{H}_{2} \mathrm{O}\right)=5.0 \mathrm{v}\right)$. If so, the fact that $\mathrm{CO}$ did not yield the bands would indicate that the dissociation energy of $\mathrm{CO}$ is greater than the energy of the upper metastable state of $\mathrm{Xe}$, namely 9.4 volts. (Energy of lower metastable state equals $8.3 \mathrm{v}$.) This appears to be direct evidence in favor of the 9.6-volt value of $\mathrm{D}(\mathrm{CO})$ as determined by Hagstrum and Tate ${ }^{1}$ from appearance potentials in the mass spectrograph, or for the $11.11 \mathrm{v}$-value obtained in a recent spectral analysis by Gaydon and Penney, ${ }^{2}$ but against the 9.14-volt value determined from certain predissociation data. ${ }^{3}$ The higher value appears to be more in accord also with thermochemical data as brought out by Hagstrum and Tate, ${ }^{1}$ and also by Asundi and Samuel. ${ }^{4}$

1 H. D. Hagstrum and J. T. Tate, Phys. Rev. 59, 365 (1941) 2 A. G. Gaydon and W. G. Penney, Proc. Roy. Soc. A183, 374 (June, 1945).

3 For a review see G. Hertzberg, Molecular Spectra and Molecular
Structure (Prentice-Hall. Inc. New York, 1939), Chap. VIII.

4 R. K. Asundi and R. Samuel, Ind. Acad. Sci. 3A, 562 (1936).

\section{Resonance Absorption by Nuclear Magnetic Moments in a Solid}

E. M. Purcell, H. C. Torrey, and R. V. Pound* Radiation Laboratory, Massachusetts Institute of Technology, Cambridge, Massachusetts December 24, 1945

$I^{\mathrm{N}}$ $\mathrm{N}$ the well-known magnetic resonance method for the determination of nuclear magnetic moments by molecular beams, ${ }^{1}$ transitions are induced between energy levels which correspond to different orientations of the nuclear spin in a strong, constant, applied magnetic field. We have observed the absorption of radiofrequency energy, due to such transitions, in a solid material (paraffin) containing protons. In this case there are two levels, the separation of which corresponds to a frequency, $\nu$, near 30 megacycles/sec., at the magnetic field strength, $H$, used in our experiment, according to the relation $h \nu=2 \mu \mathrm{H}$. Although the difference in population of the two levels is very slight at room temperature $\left(h \nu / k T \sim 10^{-5}\right)$, the number of nuclei taking part is so large that a measurable effect is to be expected providing thermal equilibrium can be established. If one assumes that the only local fields of importance are caused by the moments of neighboring nuclei, one can show that the imaginary part of the magnetic permeability, at resonance, should be of the order $h \nu / k T$. The absence from this expression of the nuclear moment and the internuclear distance is explained by the fact that the influence of these factors upon absorption cross section per nucleus and density of nuclei is just cancelled by their influence on the width of the observed resonance.

A crucial question concerns the time required for the establishment of thermal equilibrium between spins and lattice. A difference in the populations of the two levels is a prerequisite for the observed absorption, because of the relation between absorption and stimulated emission. Moreover, unless the relaxation time is very short the absorption of energy from the radiofrequency field will equalize the population of the levels, more or less rapidly, depending on the strength of this $r-f$ field. In the expectation of a long relaxation time (several hours), we chose to use so weak an oscillating field that the absorption would persist for hours regardless of the relaxation time, once thermal equilibrium had been established.

A resonant cavity was made in the form of a short section of coaxial line loaded heavily by the capacity of an end plate. It was adjusted to resonate at about $30 \mathrm{mc} / \mathrm{sec}$. Input and output coupling loops were provided. The inductive part of the cavity was filled with $850 \mathrm{~cm}^{3}$ of paraffin, which remained at room temperature throughout the experiment. The resonator was placed in the gap of the large cosmic-ray magnet in the Research Laboratory of Physics, at Harvard. Radiofrequency power was introduced into the cavity at a level of about $10^{-11}$ watts. The radiofrequency magnetic field in the cavity was everywhere perpendicular to the steady field. The cavity output was balanced in phase and amplitude against another portion of the signal generator output. Any residual signal, after amplification and detection, was indicated by a microammeter.

With the $r-f$ circuit balanced the strong magnetic field was slowly varied. An extremely sharp resonance absorption was observed. At the peak of the absorption the deflection of the output meter was roughly 20 times the magnitude of fluctuations due to noise, frequency, instability, etc. The absorption reduced the cavity output by 0.4 percent, and as the loaded $Q$ of the cavity was 670 , the imaginary part of the permeability of paraffin, at resonance, was about $3 \cdot 10^{-6}$, as predicted.

Resonance occurred at a field of 7100 oersteds, and a frequency of $29.8 \mathrm{mc} / \mathrm{sec}$., according to our rather rough calibration. We did not attempt a precise calibration of the field and frequency, and the value of the proton magnetic moment inferred from the above numbers, 2.75 nuclear magnetons, agrees satisfactorily with the accepted value, 2.7896, established by the molecular beam method.

The full width of the resonance, at half value, is about 10 oersteds, which may be caused in part by inhomogeneities in the magnetic field which were known to be of this order. The width due to local fields from neighboring nuclei had been estimated at about 4 oersteds.

The relaxation time was apparently shorter than the time ( $\sim$ one minute) required to bring the field up to the resonance value. The types of spin-lattice coupling suggested by I. Waller ${ }^{2}$ fail by a factor of several hundred to account for a time so short.

The method can be refined, in both sensitivity and precision. In particular, it appears feasible to increase the sensitivity by a factor of several hundred through a change in detection technique. The method seems applicable to the precise measurement of magnetic moments (strictly, gyromagnetic ratios) of most moderately abundant nuclei. 
It provides a way to investigate the interesting question of spin-lattice coupling. Incidentally, as the apparatus required is rather simple, the method should be useful for standardization of magnetic fields. An extension of the method in which the $r-f$ field has a rotating component should make possible the determination of the sign of the moment.

The effect here described was sought previously by Gorter and Broer, whose experiments are described in a paper $^{3}$ which came to our attention during the course of this work. Actually, they looked for dispersion, rather than absorption, in $\mathrm{LiCl}$ and $\mathrm{KF}$. Their negative result is perhaps to be attributed to one of the following circumstances: (a) the applied oscillating field may have been so strong, and the relaxation time so long, that thermal equilibrium was destroyed before the effect could be observed-(b) at the low temperatures required to make the change in permeability easily detectable by their procedure, the relaxation time may have been so long that thermal equilibrium was never established.

* Harvard University, Society of Fellows (on leave).

I Rabi, Zacharias, Millmann, and Kusch, Phys. Rev. 53, 318 (1938)

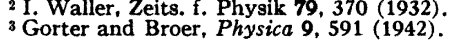

\section{The $\boldsymbol{\gamma}$-Rays of Radium D}

TSIEN SAN-TSIANG*

Laboratorie Curie, Institut du Radium, and Laboratoire de Chimie Nucléaire du College de France, Paris, France December 13, 1945

$F^{\circ}$

OR many years $\mathrm{RaD}$ was regarded as a radioactive substance which emits nuclear $\beta$-rays of very low energy and monochromatic $\gamma$-rays of quantum energy 46.7 kev. Just before the war, however, it was shown by Amaldi and Rasetti, ${ }^{1}$ using the method of selective absorption, that the radiation is complex, the well-known $46.7 \mathrm{kev}$ line being accompanied by a much weaker component of energy $43 \mathrm{kev}$. Following the publication of this result, a systematic study of the subject was started in Paris with a very strong source of $\mathrm{RaD}$ of about $100 \mathrm{mC}$, extracted previously by Professors Irène Joliot-Curie and F. Joliot. The following is a brief summary of the main experimental results, obtained in the Curie Laboratory and in the Laboratory of Nuclear Chemistry in the difficult conditions which prevailed from 1942-1945. The results deal with the detailed structure of the $\gamma$-radiation, the absolute intensity of the different lines, and the nature of $\gamma$-rays of quantum energy $46.7 \mathrm{kev}$.

In the region between 25 and $100 \mathrm{kev}$, the analysis made by the methods of selective absorption ${ }^{2}$ and crystal diffraction $^{3}$ leads to the conclusion that $\mathrm{RaD}$ emits in this region four $\gamma$-lines $(A, B, C, D)$ and two lines of $\mathrm{x}$-rays of $\mathrm{K}-83$, the corresponding energy and intensity being given in Table I.

The existence of these six different radiations is confirmed by another series of experiments in which the corresponding quantum energies are determined by measuring the true range of the photoelectrons which they project in a Wilson chamber. ${ }^{4,5}$ This method also suggested the existence of an additional weak line at about $65 \mathrm{kev}(\mathrm{x})$ and relatively intense radiations below $25 \mathrm{kev}$.

TABLE I. $\gamma$-rays from RaD.

\begin{tabular}{cccc}
\hline \hline & & $\begin{array}{c}I \\
\text { (quanta per } \\
\text { 100 disin- } \\
\text { tegrations) }\end{array}$ & Reference \\
\hline$(X)$ & $E$ (kev) & $(<0.2)$ & $(5)$ \\
$A$ & $(65 \pm 5)$ & $2.8 \pm 0.6$ & $(1,2,3,5,8)$ \\
$B$ & $46.7 \pm 0.1$ & $0.2 \pm 0.1$ & $(1,2,3,5)$ \\
$C$ & $43 \pm 1$ & $0.2 \pm 0.1$ & $(2,3,5)$ \\
$D$ & $37 \pm 1$ & $0.4 \pm 0.2$ & $(2,3,5,6)$ \\
$E$ & $32 \pm 1$ & $1.0 \pm 0.5$ & $(5,6)$ \\
$F$ & $23.2 \pm 0.6$ & $\sim 10$ & $(6,7)$ \\
\hline \hline
\end{tabular}

In order to study the region below $25 \mathrm{kev}$, experiments were made with a Wilson chamber operating at suitable low pressure. ${ }^{6}$ It was thus found that in addition to the $L$ spectrum of element 83 , there is a new $\gamma$-ray line $(E)$ of $23.2 \pm 0.6 \mathrm{kev}$. This line has thus almost exactly half the quantum energy of the principle line $(A)$ and would be confused in diffraction spectrum experiments with the second-order image of the $(A)$ line. Another very intense $\gamma$-ray line $(F)$ of $7.3 \pm 0.7 \mathrm{kev}$ has been observed in the energy region below the $L$ levels of element 83. ${ }^{6 a}$ This radiation has been observed previously by Droste ${ }^{7}$ who classified it as one of the components of the $L$ spectrum of element 83 . From the energy determinations in our present experiments, it seems more reasonable to regard it as a new $\gamma$-ray line.

In order to investigate the nature of the principle line $(A)$ we have determined the intensity of the conversion electrons of this radiation by the usual methods of magnetic $\beta$-ray spectrography and obtained the value 2.9 for the internal conversion coefficient, $N_{e} / N_{r}$ in the $L_{1}$ level.8,2 The comparison with the theoretical calculation of $\mathrm{Fisk}^{9}$ indicates that the $46.7 \mathrm{kev}$ line is most probably a quadripole radiation. For other $\gamma$-rays there is no information available about their conversion electrons ${ }^{10}$ to permit similar deduction about their nature to be made.

I should like to thank Professors I. Joliot-Curie and F. Joliot for their continuous interest and help, and Drs. Frilley, Surugue, and Ouang, and Mr. Marty for their important contributions and friendly collaboration in the course of these investigations.

* Now temporarily at H. H. Wills Physics Laboratory, Bristol, England.

1 E. Amaldi and F. Rasetti, Ricerca Scient. 10, 111 (1939).

2 Tsien San-Tsiang, Comptes rendus 216, 765 (1943); 218, 503 (1944). M. Frilley, Comptes rendus 218, 505 (1944)

5 Tsien San-Tsiang, Ann. d. Physik 19, 327 (1944). May 1945 (in press)

Tsien San-Tsiang and C. Marty, Comptes rendus, Seance of 30th July, 1945 (in press)

${ }_{6 a} \mathrm{M}$. Frilley and Tsien San-Tsiang, Comptes rendus (Jan., 1945)

7 G. von Droste, Zeits. f. Physik 84, 17 (1933).

8 Ouang Te-Tchao, J. Surugue, and Tsien San-Tsiang, Comptes rendus 217,535 (1943).

J. S. Fisk, Proc. Roy. Soc. 143, 674 (1934).

${ }^{10} \mathrm{H}$. O. W. Richardson and A. Leigh-Smith, Proc. Roy. Soc. 160 454 (1937). 